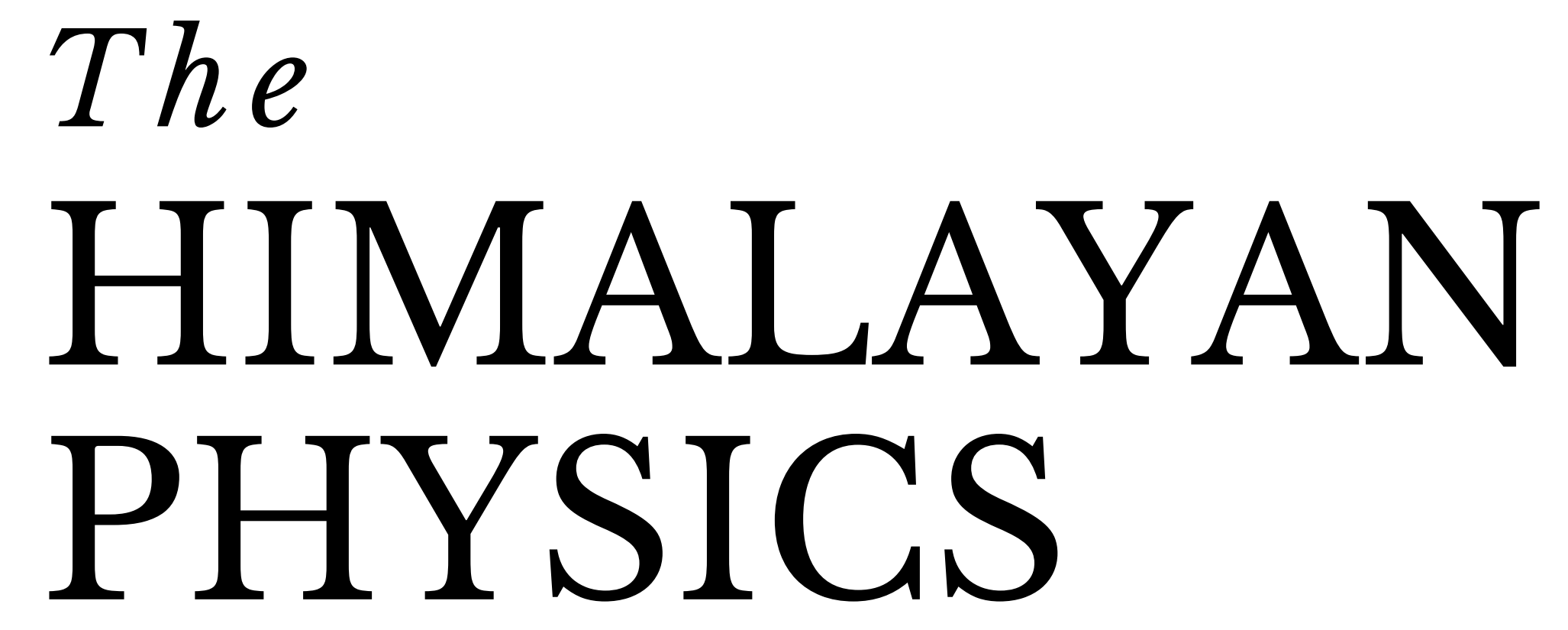

A peer-reviewed Journal of Physics

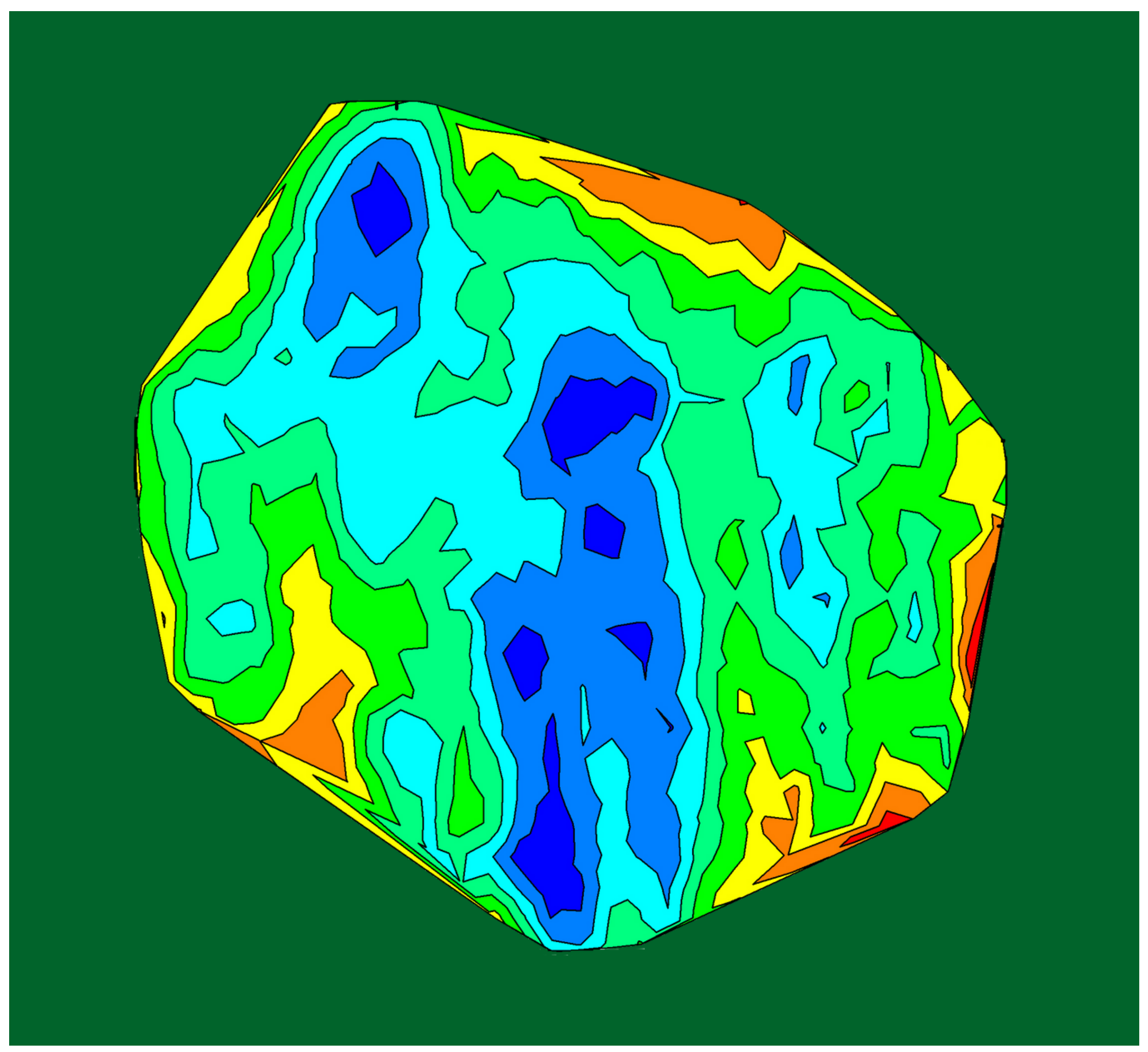

Department of Physics, Prithvi Narayan Campus, Pokhara Nepal Physical Society, Western Chapter, Pokhara 


\section{Publisher}

Department of Physics, Prithvinarayan Campus, Pokhara Nepal Physical Society, Western Chapter, Pokhara

\section{The Himalayan Physics}

Volume 9, December 2020

ISSN 2542-2545

The Himalayan Physics (HimPhys) is an open access peer-reviewed journal that publishes quality articles which make innovative contributions in all areas of Physics. HimPhys is published annually by Nepal Physical Society (Western Regional Chapter), and Department of Physics, Prithvi Narayan Campus, Pokhara. The goal of this journal is to bring together researchers and practitioners from academia in Nepal and abroad to focus on advanced techniques and explore new avenues in all areas of physical sciences and establishing new collaborations with physics community in Nepal.

\section{Chief Editor}

Kapil Adhikari

\section{Associate Editor \\ Aabiskar Bhusal}

(C)2020, Publishers. All rights reserved.

This publication is in copyright. Subject to statutory exception and to the provisions of relevant collective licensing agreements, no reproduction of any part may take place without written permission of the publishers.

Cover: Contour map of dust mass. (C) Mijas Tiwari. Printed from article in the current issue, with permission. 


\section{The HIMALAYAN PHYSICS}

A peer-reviewed Journal of Physics

\section{Chief Editor}

Kapil Adhikari

Associate Editor

Aabiskar Bhusal

\section{Publisher}

Department of Physics, Prithvi Narayan Campus, Pokhara Nepal Physical Society, Western Chapter, Pokhara 


\section{Nepal Physical Society Western Regional Chapter Pokhara, Nepal}

\section{President}

Min Raj Lamsal

Immediate Past President

Jeevan Regmi

Vice-President

Sundar Prasad Dhakal

Secretary

Ravi Karki

Treasurer

Dipak Adhikari

Joint Secretary

Sujan Lamsal

Editorial Member

Kapil Adhikari

Members

Amrit Dhakal

Laxman Thapa

Laxman Timilsina

Narayan Prasad Bhandari

Pradeep Subedi

Advisory Board

Prof. Dr. Pradip K. Bhattarai

Pabitra Mani Poudyal

Surya Bahadur G.C.

Parashu Ram Poudel

Prof. Dr. Shovakanta Lamichhane

Kul Prasad Dahal

Dr. Krishna Raj Adhikari

Ram Sajile Verma 


\section{Himalayan Physics Vol-9 (2020)}

\section{TABLE OF CONTENTS}

Metal Organic Frameworks(MOFs) as efficient carrier for targeted nanodrug delivery

R. Karki, D. Adhikari, K. Adhikari, N. Pantha

A Density Functional Theory Study on Paracetamol-Oxalic Acid Co-Crystal

P. Paudel, K.R. Adhikari, K. Adhikari

First-principles study of $\mathrm{C}$ cites vacancy defects in water adsorbed graphene

H.K. Neupane, N.P. Adhikari

Diusion of fructose in water: a molecular dynamics study

S. Bhusal, N. Pantha

Study of aecting factors of meteorological parameters on solar radiation on Pokhara P.M. Shrestha, J. Regmi, U. Joshi, K.N. Poudyal, N.P. Chapagain, I.B. Karki

Variation of mean value of velocity of ion with dierent obliqueness of magnetized plasma sheath

B.R. Adhikari, H.P. Lamichhane, R. Khanal

Study of dust properties of two far infrared cavities nearby asymptotic giant branch stars under infrared astronomical satellite maps

M. Tiwari, S.P. Gautam, A. Silwal, S. Subedi, A. Paudel, A. K. Jha

An experimental study on irradiated interface of silicon

M.R. Lamsal

Calculation of energy loss of proton beam on thyroid tumor

K. Giri, B. Paudel, B.R. Gautam

Study of noise level status at dierent rice mills in Surkhet Valley, Nepal

D.R. Paudel, H.N. Baral

Elliptically polarized laser assisted elastic electron-hydrogen atom collision and differential scattering cross-section

K. Yadav, S.P. Gupta, J.J. Nakarmi

Geodynamics of Gorkha earthquake (Mw 7.9) and its aftershocks

R.K. Tiwari and H. Paudyal 


\title{
A Density Functional Theory Study on Paracetamol-Oxalic Acid Co-Crystal
}

\author{
Punya Paudel $^{1}$, Krishna Raj Adhikari ${ }^{2 *}$, Kapil Adhikari ${ }^{1}$ \\ 1 Department of Physics, Prithivi Narayan Campus, Tribhuvan University, Pokhara, Nepal \\ 2 Department of Applied Science, Institute of Engineering, Pashchimanchal Campus, Tribhuvan University, Pokhara, \\ Nepal
}

\begin{abstract}
Paracetamol (PCA) has two well-known polymorphic forms, monoclinic (form I) and orthorhombic (form II). The parallel packing of flat hydrogen bonded layers in the metastable form II results in compaction properties superior to the thermodynamic stable form I which contains corrugated hydrogen bonded layers of molecules. In this study, the structure of Paracetamol (PCA)-Oxalic acid (OXA) co-crystal has been analyzed and found layered structure similar to PCA form II which enhance ability to form tablet. The Density Functional Theory (DFT) has been conducted to find some physicochemical properties of co-crystal. It was observed that the lattice energy of co-crystal is more than that of PCA form II showing more stability on co-crystal. The energy gap between highest occupied molecular orbital and lowest unoccupied molecular orbital (HOMO-LUMO gap) in co-crystal was found less than PCA form II showing bigger enhancement of reactivity.
\end{abstract}

Keywords: polymorph $\bullet$ monoclinic $\bullet$ orthorhombic $\bullet$ co-crystal $\bullet$ density functional theory $\bullet$ HOMO-LUMO gap

\section{Introduction}

Cocrystals are "solids that are neutral crystalline single phase materials composed of two or more different molecular and/or ionic compounds generally in a stoichiometric ratio which are neither solvates nor simple salts" [1]. The components of co-crystal interact through non covalent interactions. Most common interactions are hydrogen bonding, ionic interaction, dipole-dipole interaction and Van der Waals interaction. The resulting crystal structures from these interactions can generate physical and chemical properties such as melting point, solubility, chemical stability and mechanical properties that differ from properties of individual components [2]. The application of co-crystal is relevant to production of energetic material, pharmaceuticals and other compounds. The most widely studied and used application of co-crystal engineering is in the drug development and more specially the formation, design and implementation of an active pharmaceutical ingredient (API). Pharmaceutical co-crystal is crystalline material consisting of an API and one or more unique co-crystal formers. Pharmaceutical co-crystals are becoming popular in recent years due to their potential to improve the physicochemical properties

\footnotetext{
* Corresponding Author: adhikari.krishnaraj@gmail.com
} 
of drug substances such as solubility, dissolution rate, stability, hygroscopicity, and compressibility without alternating their pharmacological behavior. Developing superior physiochemical properties of API while holding the properties of the drug molecule itself constant is the principle idea of pharmaceutical co-crystal engineering.

Paracetamol is widely used over-the-counter analgesic (pain reliever) and antipyretic (fever reducer). Paracetamol is also known as acetaminophen, chemically named N-acetyl-p aminophenol. It has benzene ring core which is substituted by one hydroxyl group and the acetamide group in the para $(1,4)$ pattern [3]. It was first made in 1877 [4]. It is the most commonly used medicine for pain and fever in both the US and Europe. It is also on the World Health Organization's List of Essential Medicines, which lists the most effective as well as safe medicines for our health.

Oxalic acid is a crystalline organic compound that is widely used as an acid rinse in laundries, where it acts as an agent to remove rust and ink stains because it converts most insoluble iron compounds into a soluble complexion. It is also used to prepare many commercials for removing scale from automobiles radiation. Oxalic acid can be naturally found in many foods but excessive ingestion of it can be toxic and prolonged skin contact can be dangerous too. Oxalic acid is a carboxylic acid with the formula $\mathrm{C}_{2} \mathrm{H}_{2} \mathrm{O}_{4}$. Oxalic acid was first prepared synthetically in 1776 however its usual form is that of the crystalline hydrate, $(\mathrm{COOH})_{2} \cdot 2 \mathrm{H}_{2} \mathrm{O}$ known as a constituent of wood sorrel as early as the 17th century.

Paracetamol has two well-known polymorphic forms; the monoclinic form I is stable and orthorhombic form II is metastable. The metastable form II has parallel packing of flat hydrogen bonded layers while the thermodynamic stable form I contains corrugated hydrogen bonded layers of molecules. The parallel packing of flat hydrogen bonded layers in the form II results in compaction properties superior to the form I [5] [6]. Although form II would, therefore, be preferred in an industrial tableting process, its lower thermodynamic stability prevents harvesting its use commercially. As a result, Paracetamol tablet on market consists of thermodynamic stable form I accompanied by a large loading of binder that prevents chipping and disintegration. Consequently, the development of a layered, thermodynamically stable, and pharmaceutically acceptable form of Paracetamol with properties similar to form II is an interesting challenge to pharmaceutical crystal engineering. The challenge is further increased by the lack of acidic or basic functionalities on the Paracetamol molecule, preventing the synthesis of salts as alternative solid forms [7]. Hence co-crystallization can be used as strategy to generate layered solid forms of Paracetamol that would be thermodynamically stable and exhibit tablet-forming properties resembling form II.

As suitable co-crystal formers for the construction of a layered structure, we have to select molecules of comparable size to Paracetamol that have, or can readily adopt, a flat shape [8]. Co-crystallization of Paracetamol with co-crystal former such as Phenazine, Oxalic acid, Theophylline, Naphthalene can be studied for the compaction properties which are generally recognized as safe (GRAS) compounds. Among these compounds, Oxalic acid is relatively cheap and is also small which is good for modeling. Karki, Fri`šs ci cic, F'abi an, Peter, Day and Jones studied the co-crystal of Paracetamol with Oxalic acid and observed the resultant co-crystal with compaction 
properties superior to form I and similar to form II; while form I could not be compressed into a tablet without extensive loading of binders, co-crystallization product readily formed tablets [9]. Thus the main objective of this work is to check stability of this Paracetamol-Oxalic acid co-crystal.

In this work, the structure of co-crystal has been analyzed and compared with form II to study its observed compaction properties. Then the stability of co-crystal has been checked by calculating lattice energy and compared with form II to check whether it is more stable than form II or not. In addition we have also calculated the reactivity of co-crystal by calculating HOMO-LUMO gap and compared with form II. Here we used HOMO-LUMO gap instead of band gap because our system is molecular solid and molecular orbital energy can in principle be calculated exactly, including all particle interaction, while Band theory uses approximations based on the periodicity of the lattice in extended systems, and ignores many contributions to the specific energy of individual particles.

We have used first principles methods for our calculation. Obtaining exact solution directly of our system is very complicated and almost insoluble, in order to adopt easier and simpler procedure, certain approximation need to be applied. For the low computational cost with arbitrary accuracy, we have used Density Functional Theory (DFT) for our calculation. DFT is the most commonly used formalism in first principles method, applied for studying the structure and properties of condensed matter related to the electronic ground state of the system, for solving many electron problem. According to Density Functional Approximation, electron density of any system determines all the ground state properties of the system. So electron density can be used as functional and all other ground state properties can be calculated.

In the following section, we are going to have an overview on computational details of our work. Then, we include the main findings of our present work where we analyze the findings and provide the reasons behind them and take a glimpse of the work we performed.

\section{Computationals Methods}

We have used the technique of DFT to calculate the lattice energy and HOMO-LUMO gap from which we can find stability and reactivity of crystal respectively. Here, we used Gaussian09 software for DFT calculation with functional M06 and basis set 6-311G (2d, 2p). GaussView6 software was used for graphical user interface to prepare input for submission to Gaussian and to examine graphically the output that Gaussian produces. For the input, we used Crystallographic Information File (CIF). We took only single layer and neglected the interaction between layers which is very less in comparison with intermolecular hydrogen bond within a single layer. Due to computational cost, we took cluster consisting up to few molecule only. We used monomer, dimer and cluster up to five molecules. The lattice energy is the energy of formation of a crystal from the isolated molecules in gas phase. It is calculated by summing all non-bonded interactions for the entire, perfect crystal. In this study, cluster method has been applied to predict the lattice energy of co-crystal and form II. Here we have calculated energies 
of formation of clusters by subtracting energies of clusters from sum of energies of each molecules consisting of clusters. It gives the qualitative idea about lattice energy. Although we took clusters consisting up to only five molecules, comparing the energies of formation allows us to compare the lattice energies of co-crystal and form II, more the energy of formation, more lattice energy. We have done energy calculation of each monomers, dimers and crystal clusters with same parameters in Gaussian. From the output files of energy calculation of clusters, we calculated HOMO-LUMO gap of each cluster by subtracting energy of HOMO from energy of LUMO.

\section{Result and Discussion}

\subsection{Structure Analysis}

To study the observed compaction properties of PCA-OXA co-crystal, we have analyzed the structure of it and compared with PCA form II. Crystal structure of PCA form I, PCA form II, PCA-OXA co-crystal have been shown in figures 1, 2 and 4 respectively. The clusters containing few molecules within single layer of form II and co-crystal have been shown in figures 3 and 5 respectively.

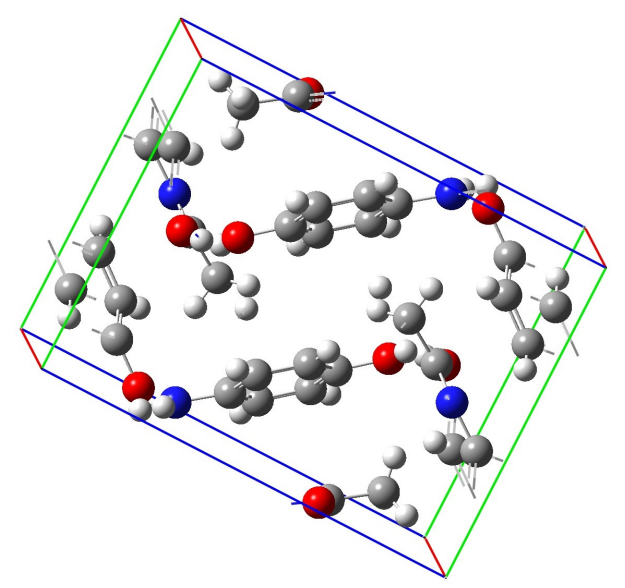

Figure 1. PCA form I consisting of corrugated hydrogen bonded layers of molecules. The crystal structure parameters are $a=6.9800 \AA, b=8.91500 \AA, A=11.56600 \AA$ and $\beta=98.5400$ (P21/a group) 


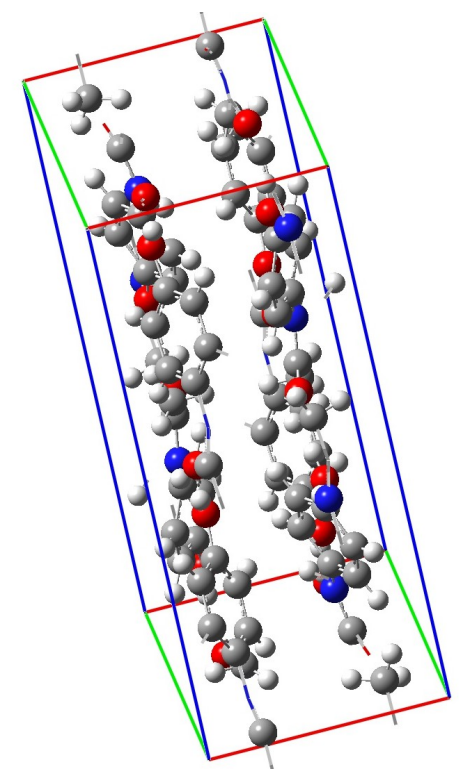

Figure 2. PCA form II consisting of parallel packing of flat hydrogen bonded layers of molecules. The crystal structure parameters are $a=7.20280 \AA, \mathrm{b}=11.77640 \AA, c=17.16770 \AA$ and $\beta=90.0000$ (Pcab group)

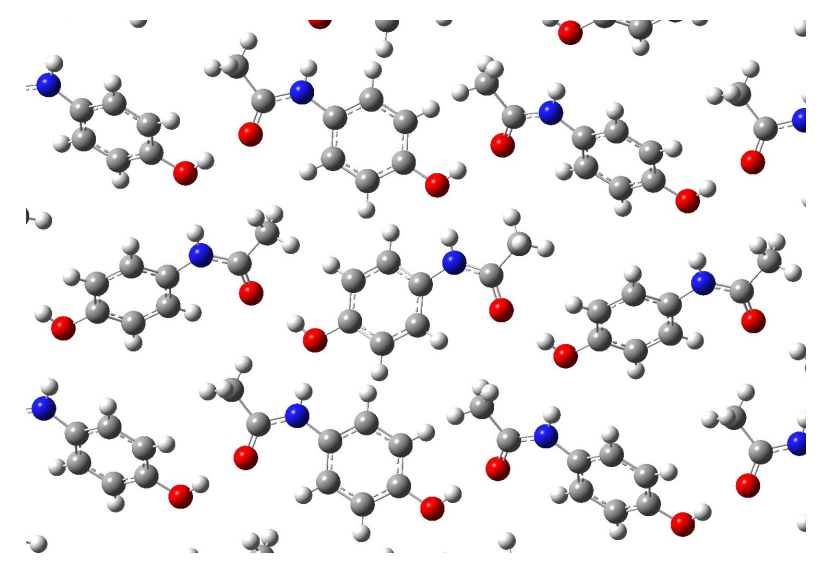

Figure 3. PCA form II single layer. PCA acts as two fold hydrogen bond donor through amide (NH) and phenol $(\mathrm{OH})$ and two fold accepter via carbonyl $(\mathrm{CO})$ and phenol $(\mathrm{OH})$ group. Thus each PCA molecule is hydrogen bonded with four neighboring PCA molecules. 


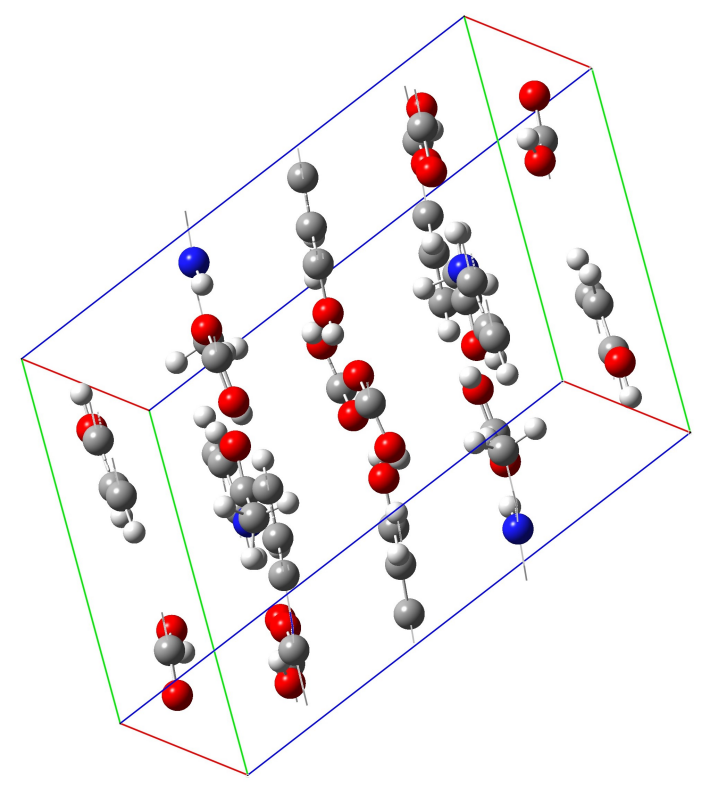

Figure 4. PCA-OXA co-crystal consisting of parallel packing of flat hydrogen bonded layers of molecules. The crystal structure parameters are $a=5.16384 \AA$ A $b=11.73230 \AA, c=17.59340 \AA$ and $\beta=94.3285(\mathrm{P} 21 / \mathrm{c}$ group)

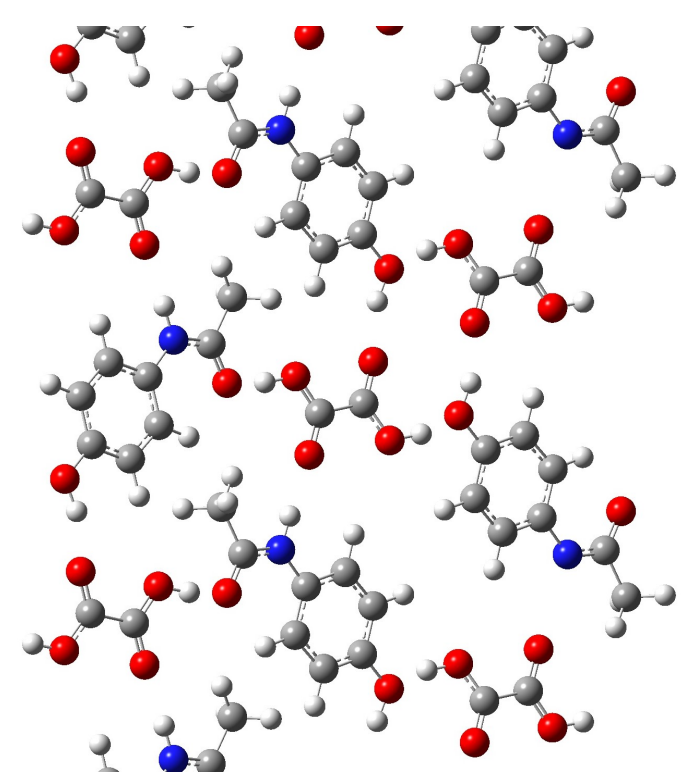

Figure 5. PCA-OXA co-crystal single layer. PCA acts as two fold hydrogen bond donor through amide (NH) and phenol $(\mathrm{OH})$ and two fold accepter via carbonyl $(\mathrm{CO})$ and phenol $(\mathrm{OH})$ group. Thus each PCA molecule is hydrogen bonded with four neighboring OXA molecule and vice versa.

Structural analysis of crystal structure of PCA-OXA confirmed that co-crystal is composed of hydrogen bonded PCA-OXA molecule which form hydrogen bonded parallel layer similar to form II. As like form II, PCA acts as two fold hydrogen bond donor through amide $(\mathrm{NH})$ and phenol $(\mathrm{OH})$ and two fold accepter via carbonyl $(\mathrm{CO})$ and phenol $(\mathrm{OH})$ group in each layer. Thus each PCA molecule is hydrogen bonded with four neighboring 
OXA molecule and vice versa. The outcome is layered structure achieved by two dimensional tiling of 0D motifs of PCA-OXA similar to form II. Thus the parallel packing of flat hydrogen bonded layers of molecules is the reason for the observed compaction properties of PCA-OXA co-crystal as in the case of PCA form II. The layered structure of co-crystal provides the explanation for the observed enhanced ability to form tablets.

\subsection{Stability and Chemical Reactivity}

\subsubsection{Lattice energy}

To check the stability of PCA-OXA co-crystal and to know whether this co-crystal is more stable than form II of PCA or not, we have calculated energies of formation of clusters of co-crystal and form II. The calculated energies of formation of PCA-OXA and PCA form II clusters have been shown in Table 1.

Table1: List of molecular clusters with energies of formation. The first column lists the name of clusters. The second column gives the calculated energies of formation.

\begin{tabular}{|c|c|}
\hline Name & Energy of formation (eV) \\
\hline PCA-OXA dimer & 0.14 \\
\hline PCA-OXA 1:4 (five molecules) cluster & 0.36 \\
\hline PCA-OXA 4:1 (five molecules) cluster & 0.41 \\
\hline PCA form II dimer & 0.12 \\
\hline PCA form II (five molecules) cluster & 0.31 \\
\hline
\end{tabular}

The energies of formation are more in PCA-OXA clusters than in PCA form II clusters which shows more lattice energy of co-crystal than form II. More lattice energy implies more stability of co-crystal. Thus we found that this co-crystal of PCA-OXA has similar structure like form II of PCA but more stable. Although form I of PCA is stable, it is not compact enough to make tablet without large loading of binder that prevents chipping and disintegration but co-crystal is stable as well as compact. Hence, co-crystal of PCA-OXA can be the better solution to the todays marketed PCA.

\subsubsection{HOMO-LUMO gap}

To find the reactivity of PCA-OXA co-crystal and to know whether it is more reactive than form II of PCA or not, we have calculated HOMO-LUMO gaps of clusters of co-crystal and form II. The HOMO-LUMO gaps of PCA-OXA and PCA form II clusters have been shown in Table 2.

Table 2: List of molecular clusters with HOMO-LUMO gaps. The first column lists the name of clusters. The next two columns give the energy of HOMO $\left(E_{H}\right)$ and LUMO $\left(E_{L}\right)$. The last column gives the calculated the HOMO-LUMO gap $\left(E_{L}-E_{H}\right)$.

\begin{tabular}{|c|c|c|c|}
\hline Name & $E_{H}(\mathrm{eV})$ & $E_{L}(\mathrm{eV})$ & $E_{L}-E_{H}(\mathrm{eV})$ \\
\hline PCA-OXA dimer & -5.6993 & -2.5086 & 3.19 \\
\hline PCA-OXA 1:4 (five molecules) cluster & -6.1315 & -2.6444 & 3.49 \\
\hline PCA-OXA 4:1 (five molecules) cluster & -5.5704 & -1.9934 & 3.58 \\
\hline PCA form II dimer & -5.9130 & -0.7502 & 5.16 \\
\hline PCA form II (five molecules) cluster & -5.5671 & -1.2029 & 4.36 \\
\hline
\end{tabular}

The HOMO-LUMO gaps are smaller in PCA-OXA clusters than PCA form II clusters. Smaller the energy gap, more easily molecules electron can excite; electron can jump to unoccupied molecular orbital by application 
of small energy. Thus PCA-OXA co-crystal is more reactive than PCA form II. More reactive drugs can do metabolism faster to form metabolites which are less chemically active than original molecules and leave the body faster which may be helpful for reducing the side effects of drugs.

\section{Conclusion}

Although the form II of Paracetamol has parallel packing of flat hydrogen bonded layers which results in compaction properties superior to form I containing corrugated hydrogen bonded layers of molecule, it is not stable enough. Co-crystallization with oxalic acid gives better stability as well as reactivity with structure similar to form II which helps to make better tablet than form I. Hence, Co-crystal of Paracetamol with Oxalic acid can be the better solution to the todays marketed Paracetamol which consists of stable form I accompanied by large loading of binder that prevent chipping and disintegration.

\section{References}

[1] Aitipamula S, Banerjee R, Bansal AK, Biradha K, Cheney ML, Choudhury AR, et al. Polymorphs, salts, and cocrystals: what's in a name? Crystal growth \& design. 2012;12(5):2147-2152.

[2] Braga D, Grepioni F, Maini L, Polito M. Crystal polymorphism and multiple crystal forms. Molecular networks. 2009:87-95

[3] Bales J, Nicholson J, Sadler P. Two-dimensional proton nuclear magnetic resonance" maps" of acetaminophen metabolites in human urine. Clinical chemistry. 1985;31(5):757-762.

[4] Mangus BC, Miller MG. Pharmacology application in athletic training. FA Davis; 2005.

[5] Kachrimanis K, Fucke K, Noisternig M, Siebenhaar B, Griesser UJ. Effects of moisture and residual solvent on the phase stability of orthorhombic paracetamol. Pharmaceutical research. 2008;25(6):1440-1449.

[6] Beyer T, Day GM, Price SL. The prediction, morphology, and mechanical properties of the polymorphs of paracetamol. Journal of the American Chemical Society. 2001;123(21):5086-5094.

[7] Trask A, Haynes D, Motherwell W, Jones W. Electronic Supplementary Information for: "Screening for Crystalline Salts via Mechanochemistry". Acta Crystallogr. 2001;57:459.

[8] Childs SL, Stahly GP, Park A. The salt- cocrystal continuum: the influence of crystal structure on ionization state. Molecular pharmaceutics. 2007;4(3):323-338.

[9] Karki S, Friščić T, Fabian L, Laity PR, Day GM, Jones W. Improving mechanical properties of crystalline solids by cocrystal formation: new compressible forms of paracetamol. Advanced materials. 2009;21(38-39):39053909 . 